reaction-single strand conformation polymorphism (PCR-SSCP) coupled with sequencing analysis was used as the screening method for TAGAP variants.

Results Statistical significant difference was observed in Rs212389 alleles' distribution between RA patients and controls $(p=0.018$, $\mathrm{OR}=0.646$, 95\%CI: 0.449-0.930). No statistical significant difference was revealed in distribution of variants Rs394581 and Rs182429 between the studied groups.

Conclusions Our results confirm that the polymorphism Rs212389 confers the risk to RA liability in Europeans. However, more studies in larger groups of patients and controls and of multiple origins are needed as to confirm and increase the power of the suggested association.

\section{A7.20 RESPONSE TO INFLIXIMAB THERAPY CAN BE PREDICTED USING DISTINCT, NON-OVERLAPPING GENE PANELS OF PERIPHERAL BLOOD GENE EXPRESSION IN RHEUMATOID ARTHRITIS AND CROHN'S DISEASE}

doi:10.1136/annrheumdis-2013-203221.20

'Zoltán Szekanecz, ${ }^{2}$ Bertalan Meskó, 2.3Szilárd Poliska, 'Andrea Váncsa, ${ }^{4}$ Károly Palatka,

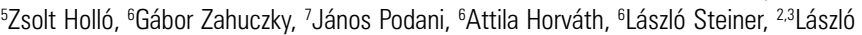
Nagy. ${ }^{~}$ Department of Rheumatology, Institute of Medicine; ${ }^{2}$ Department of Biochemistry and Molecular Biology; ${ }^{3}$ Clinical Genomics Center; ${ }^{4}$ Department of Gastroenterology, Institute of Medicine; University of Debrecen, Medical and Health Science Center, Debrecen, Hungary; ${ }^{5}$ EGIS Pharmaceuticals PIc., Budapest, Hungary; ${ }^{6} U D-G e n o M e d$, Ltd., Debrecen, Hungary; 'Institute of Biology, Loránd Eötvös University, Budapest, Hungary

Background Biological therapies targeting tumour necrosis factor $\alpha$ (TNF- $\alpha)$ have been widely used to treat chronic inflammatory disorders including rheumatoid arthritis (RA) and Crohn's disease (CD). As these treatment modalities are rather expensive, there is a need for biomarkers that may predict therapeutic responses. As RA and $\mathrm{CD}$ have similar pathogenic background, one may expect to detect overlapping gene panels in predicting the response to the same therapy by the same approach. There have been very few studies using simultaneous pharmacogenomic approach in two diseases.

Methods In this study, we performed peripheral blood global gene expression profiling followed by Canonical Variates Analysis (CVA) in $\mathrm{CD}$ and RA to identify gene sets that can differentiate responders from non-responders for infliximab therapy; and validated the results in an independent cohort by RT-OPCR

Results In CD, global gene expression analysis of samples obtained at baseline resulted in a list of 48 probe sets differentiating responders from non-responders. Comparing baseline and week 2 samples resulted in a list of five genes including AOP9, IGKC, MGAM, MMP8 and TNFAIP6 that exert expression changes upon treatment. Similarly, in RA, analysis of baseline samples resulted in a list of 30 probe sets that differentiated responders from non-responders. Expression of 3 genes; AOP9, IGJ and TNFAIP6 showed significant difference between baseline and week 2 samples. Results were validated by RT-QPCR on independent patient cohorts in both diseases. CVA analysis yielded to 15 and 12 genes in RA and CD, respectively, showing the best discriminatory power between responders and non-responders in both diseases, however there was no overlap between these two gene lists.

Conclusions We provided two pieces of proof of concept evidence showing that 1) peripheral blood gene expression profiles are suitable for determining gene panels with the highest discriminatory power in order to differentiate responders and non-responders in a patient cohort in $\mathrm{CD}$ and $\mathrm{RA}$; and 2) distinct, non-overlapping gene panels are required for the prediction of the responder status in CD and RA despite the fact that these conditions have similar pathogenic background. Application of such gene panels could solve unmet needs in the clinical settings by determining specific responses to expensive biological therapies.

\section{A7.21 SUPPRESSION OF HDAC5 EXPRESSION BY INFLAMMATORY CYTOKINES IS REOUIRED TO PROMOTE CXCL CHEMOKINE PRODUCTION IN RA FLS}

doi:10.1136/annrheumdis-2013-203221.21

${ }^{1} \mathrm{C}$ Angiolilli, 'AM Grabiec, 'LGM van Baarsen, 1,2PP Tak, 'DL Baeten, 'KA Reedquist. ${ }^{1}$ Department of Experimental Immunology and Department of Clinical Immunology and Rheumatology, Academic Medical Center, University of Amsterdam, Amsterdam, The Netherlands; ${ }^{2}$ Currently also: GlaxoSmithKline, Stevenage, UK

Background and Objectives Histone deacetylases (HDACs) are important regulators of gene expression and protein function in the immune system. HDAC inhibitors (HDACi) display antiinflammatory properties in animal and in vitro models of rheumatoid arthritis (RA), as well as initial safety and efficacy in the treatment of systemic onset juvenile idiopathic arthritis. However, as most of the currently available HDACi display little selectivity or specificity for class I (HDAC 1-3, 8) and class II (HDAC 4-6, 9, 10) HDACs, the role of specific HDACs in RA is unclear. Here we examined the relationship between HDACs and inflammation in RA synovial tissue and fibroblast-like synoviocytes (FLS).

Materials and Methods RNA was isolated from arthroscopic synovial biopsies from 19 RA patients. MMP-1, TNF $\alpha$, IL-6, and HDAC 1-10 expression was measured by quantitative PCR (qPCR). RA FLS were stimulated with IL-1 $\beta$, TNF $\alpha$ and LP, S and HDAC expression was measured by qPCR RA FLS were transduced with adenovirus encoding control GFP or GFP-HDAC5 or transfected with control siRNA or siRNA targeting HDAC5. Effects of HDAC5 modulation on RA FLS gene expression were analysed by custom qPCR array.

Results Positive correlations were observed between RA synovial tissue expression of TNF $\alpha$ and HDAC1 $(R=0.651, P=0.003)$ HDAC2 $(\mathrm{R}=0.523, \mathrm{P}=0.022)$ and HDAC3 $(\mathrm{R}=0.570, \mathrm{P}=0.011)$ and between MMP-1 and HDAC1 $(\mathrm{R}=0.501, \mathrm{P}=0.029)$ and HDAC2 $(\mathrm{R}=0.512, \mathrm{P}=0.025)$. A significant negative correlation was observed between synovial tissue expression of IL-6 and HDAC5 $(\mathrm{R}=-0.477, \mathrm{P}=0.039)$ and between clinical parameters of disease activity and HDAC5 (CRP: $\mathrm{R}=-0.664, \mathrm{P}=0.007$; $\mathrm{ESR}: \mathrm{R}=-0.556$, $\mathrm{P}=0.013$ : DAS28: $\mathrm{R}=-0.567, \mathrm{P}=0.011)$. HDAC5 mRNA expression was significantly and selectively reduced after RA FLS stimulation with TNF $\alpha$ and IL-1 $\beta$, but not LPS. Of 84 genes regulated in RA FLS by IL- $1 \beta$ or TNF $\alpha$, mRNA expression of CXCL9, CXCL10, and CXCL11 was selectively upregulated following silencing of HDAC5 expression. Conversely, mRNA expression of these chemokines was suppressed by overexpression of HDAC5 in RA FLS.

Conclusions RA synovial expression of HDAC 1 and 2, but not class II HDACs, positively correlates with local inflammatory mediators, while HDAC5 expression negatively correlates with IL-6 mRNA expression and with disease activity. HDAC5 mRNA is decreased after inflammatory stimulation, and silencing of HDAC5 leads to an increase of CXCL chemokine expression in RA. FLS, an effect reversed by HDAC5 overexpression. Our results suggest a protective role for HDAC5 in RA, and that HDACi which fail to target HDAC5 may be more promising for therapeutic applications.

\section{A7.22 THE ETS1 TRANSCRIPTION FACTOR MAY PREDISPOSE TO RHEUMATOID ARTHRITIS SUSCEPTIBILITY}

doi:10.1136/annrheumdis-2013-203221.22

A Chatzikyriakidou, PV Voulgari, AA Drosos. Rheumatology Clinic, Department of Internal Medicine, Medical School, University of loannina, Greece 